\title{
Impaired sleep quality and sleep duration in smokers-results from the German Multicenter Study on Nicotine Dependence
}

\author{
Stefan Cohrs ${ }^{1,2,3}$, Andrea Rodenbeck ${ }^{2,3}$, Dieter Riemann ${ }^{4}$, Bertram Szagun ${ }^{5}$, Andreas Jaehne ${ }^{4}$, \\ Jürgen Brinkmeyer ${ }^{6}$, Gerhard Gründer ${ }^{7}$, Thomas Wienker ${ }^{8}$, Amalia Diaz-Lacava ${ }^{8,15}$, \\ Arian Mobascher', Norbert Dahmen', Norbert Thuerauf ${ }^{10}$, Johannes Kornhuber ${ }^{10}$, Falk Kiefer ${ }^{\prime \prime}$, \\ Jürgen Gallinat ${ }^{12}$, Michael Wagner ${ }^{13}$, Dieter Kunz ${ }^{1,16}$, Ulrike Grittner ${ }^{14}$ \& Georg Winterer ${ }^{15}$ \\ Department of Sleep Medicine, St. Hedwig Clinic Berlin, Berlin, Germany', Institute of Physiology, Sleepresearch \& Clinical Chronobiology, Charité, Berlin, Germany², \\ Department of Psychiatry and Psychotherapy, Georg-August University Göttingen, Göttingen, Germany ${ }^{3}$, Department of Psychiatry and Psychotherapy, University \\ of Freiburg, Freiburg, Germany4, University of Applied Sciences, Ravensburg-Weingarten, Germany, Department of Psychiatry, Heinrich-Heine University \\ Düsseldorf, Düsseldorf, Germany ${ }^{6}$, Department of Psychiatry, Psychotherapy, and Psychosomatics, RWTH Aachen University, Aachen, Germany ${ }^{7}$, Institute of \\ Medical Biometry, Informatics and Epidemiology, University of Bonn, Bonn, Germany ${ }^{8}$, Department of Psychiatry, Johannes Gutenberg University, Mainz, Germany ${ }^{9}$, \\ Department of Psychiatry, Friedrich-Alexander University, Erlangen-Nuernberg, Germany ${ }^{10}$, Department of Addictive Behavior and Addictive Medicine, Central \\ Institute of Mental Health, Mannheim, Germany"', Department of Psychiatry, Charité University Hospital, Berlin, Germany'2, Department of Psychiatry, University \\ of Bonn, Bonn, Germany'3, Department of Biostatistics and Clinical Epidemiology, Charité- University Medicine, Berlin, Germany'4, Cologne Center for Genomics \\ (CCG), Cologne University, Cologne, Germany ${ }^{15}$ and German Heart Institute, Berlin, Germany'16
}

\section{ABSTRACT}

Cigarette smoking is a severe health burden being related to a number of chronic diseases. Frequently, smokers report about sleep problems. Sleep disturbance, in turn, has been demonstrated to be involved in the pathophysiology of several disorders related to smoking and may be relevant for the pathophysiology of nicotine dependence. Therefore, determining the frequency of sleep disturbance in otherwise healthy smokers and its association with degree of nicotine dependence is highly relevant. In a population-based case-control study, 1071 smokers and 1243 nonsmokers without lifetime Diagnostic and Statistical Manual of Mental Disorders, Fourth Edition, Axis I disorder were investigated. Sleep quality (SQ) of participants was determined by the Pittsburgh Sleep Quality Index. As possible confounders, age, sex and level of education and income, as well as depressiveness, anxiety, attention deficit hyperactivity, alcohol drinking behaviour and perceived stress, were included into multiple regression analyses. Significantly more smokers than non-smokers $(28.1 \%$ versus $19.1 \%$; $P<0.0001)$ demonstrated a disturbed global SQ. After controlling for the confounders, impaired scores in the component scores of sleep latency, sleep duration and global SQ were found significantly more often in smokers than non-smokers. Consistently, higher degrees of nicotine dependence and intensity of smoking were associated with shorter sleep duration. This study demonstrates for the first time an elevated prevalence of sleep disturbance in smokers compared with non-smokers in a population without lifetime history of psychiatric disorders even after controlling for potentially relevant risk factors. It appears likely that smoking is a behaviourally modifiable risk factor for the occurrence of impaired SQ and short sleep duration.

Keywords Case-control, nicotine dependence, Pittsburgh Sleep Quality Index population based, sleep, smoking intensity.

Correspondence to: Stefan Cohrs, Abt. für Schlafmedizin, St. Hedwig-Krankenhaus, Charité Berlin, Große Hamburger Str. 5-11, 10115 Berlin, Germany. E-mail: stefan.cohrs@charite.de

\section{INTRODUCTION}

Tobacco use is one of the main risk factors for a number of chronic diseases, including cancer, lung diseases and cardiovascular diseases. Continuing smokers die up to 10 years earlier than lifelong non-smokers (Doll et al. 2004).
Cessation of smoking at the age of 30 or even 40 years on the other hand may gain up to 10 years of life expectancy (Doll et al. 2004). Even so, long-lasting tobacco use is common throughout the world. One of the reasons for the adherence to smoking is that chronic nicotine consumption is usually coupled with physical and 
psychological dependence. Nicotine withdrawal symptoms may include the craving for tobacco, dysphoria, difficulty concentrating, depression, fear, anhedonia, irritability or restlessness, increased appetite and sleep disturbances (Hughes, Higgins \& Bickel 1994; American Psychiatric Association 2000). Additionally, nicotine itself has arousing effects and smoking is reported to be associated with sleep disturbance. Although the health consequences of smoking are well documented, the influence of nicotine consumption, nicotine withdrawal and substituted nicotine on sleep has not been extensively studied (Jaehne et al. 2009).

Recent research strongly supports the assumption that both smoking and smoking cessation negatively affect sleep. Preliminary data indicate that nicotine use is coupled with an increased frequency of insomniac complaints during active smoking, while nicotine withdrawal and nicotine replacement therapy further increase sleep disturbances (Colrain, Trinder \& Swan 2004; Jaehne et al. 2009). In a large longitudinal cohort study, male and female smokers were reported to suffer from difficulties getting to sleep and from non-restorative sleep much more frequently than non-smokers, whereas they did not differ in difficulties getting back to sleep, repeated awakenings and early morning awakenings (Wetter \& Young 1994). This was mainly confirmed by Phillips \& Danner (1995). In Japanese civil servants, global sleep quality was found to be significantly worse in male ex-smokers versus non-smokers and in female current smokers versus non-smokers. Additionally, male current smokers smoking 21-40 cigarettes/day and ex-smokers presented a significantly increased prevalence of poor sleep, but in women, only current smokers were associated with a high prevalence of poor sleep $(\mathrm{Hu}$ et al. 2007). In a sample of 484 adolescents and adults aged 14-84 years, cigarette smokers were significantly more likely than non-smokers to report problems going to sleep and also problems staying asleep (Phillips \& Danner 1995). Others found a reduced sleep duration in smoking adults, whereas sleep quality was hardly affected by smoking status (Palmer, Harrison \& Hiorns 1980). A broader range of sleep disturbance was observed in smoking pregnant women. Smoking increased the odds of sleep disturbances, such as subjective insufficient sleep, difficulty in initiating or maintaining sleep, early morning awakening, short sleep duration and excessive daytime sleepiness in a large group of pregnant Japanese women by a factor of around two (Kaneita et al. 2005). Adding to the diversity of findings, one large prospective longitudinal study of the natural history of obstructive lung disease found no association between smoking status and the prevalence of sleep disturbance within a control group not suffering from respiratory disease (Klink \& Quan 1987).
Some of the variability of these results may stem from the diversity of populations studied. Individual factors such as certain personality-related traits (e.g. impulsivity and diminished stress tolerance) or psychiatric disorders (e.g. schizophrenia, depression, anxiety disorder and attention deficit disorder) are well acknowledged to considerably increase risk for smoking initiation and nicotine dependence (ND) (Elkins, McGue \& Iacono 2007; Krishnan-Sarin et al. 2007; Aklin et al. 2009; O'Loughlin et al. 2009; Trosclair \& Dube 2010; Winterer 2010) and at the same time these factors are well known to be associated with sleep disturbance (Benca et al. 1992; Tsuno, Besset \& Ritchie 2005; Peterson \& Benca 2006; Cohrs 2008). Therefore, it remains unclear to what extent the described sleep disturbances in smokers are due to their smoking or related to an underlying co-morbid condition.

Additionally, very few studies considered the influence of the amount of daily smoked cigarettes on sleep. Palmer et al. reported a significant decrease in the mean sleep duration with increasingly heavy smoking (Palmer et al. 1980), whereas Riedel et al. (2004) reported to the contrary that after controlling for demographic, health, psychological and behavioural variables, light smoking ( $<15$ cigarettes per day), but not heavier smoking, was associated with self-reported chronic insomnia and reduced sleep diary total sleep time and time in bed (Riedel et al. 2004). In addition to these contradictory results with regard to the influence of the number of daily smoked cigarettes on sleep in smokers, to the best of our knowledge, no information is available regarding the influence of the degree of ND, degree of smoking urges and objective measures of nicotine consumption including exhaled CO and cotinine levels. Additionally, no study has used a validated questionnaire to evaluate sleep disturbance.

Over the last decade, sleep disturbance has been identified to play an important role in the pathophysiology of a number of physical and mental disorders. To further our knowledge about a modifiable risk factor for sleep disturbance, cigarette smoking and vice versa, the association of sleep disturbance with smoking could be a helpful step to reduce public health burden due to both cigarette smoking and sleep disturbance.

The German Multicenter Study on Nicotine Dependence is a population-based case-control study of smokers and never smokers addressing ND with the collection of a wide range of environmental, psychosocial and neurobiological phenotypes in subjects free of potentially confounding psychiatric disorders (Lindenberg et al. 2011).

The first aim of this report is the analysis of a possible association between ND and sleep disturbance. An additional analysis will test whether such an association still exists after controlling for relevant confounding variables such as age, sex, level of education and income, attention 
deficit hyperactivity disorder (ADHD), depressiveness, alcohol consumption and perceived stress. Furthermore, it will be tested whether degree of ND, number of cigarettes smoked per day, smoking urges, blood cotinine levels and exhaled CO have an influence on different aspects of sleep quality including sleep duration.

\section{METHODS}

\section{Study design}

The German Multicenter Study on Nicotine Dependence collected a wide range of parameters in order to determine possible neurobiological phenotypes. For a detailed description of this study, see Lindenberg et al. (2011). Briefly, data collection was conducted from 2007 to 2009 in seven recruitment centers throughout Germany. In order to approach 'population representativeness', all probands of the case-control sample of current smokers and never smokers were randomly selected from the local general population at each site via official local residents' registers and contacted by letter with an invitation to participate in the study. Across centers, approximately 55000 subjects were contacted by letter (10\% responded by phone). Only subjects who met the inclusion/ exclusion criteria were allowed to participate in the study. Inclusion criteria were age 18-65 years, current smoker (also occasional smoker with a minimum of seven cigarettes per week/one cigarette per day) or never smoker (maximum of 100 cigarettes/lifetime), grandparents of probands were required to be born in Germany or in a country adjacent to Germany, mother-tongue level German and each proband must have been personally approached by letter (via official local residents' registers). Exclusion criteria were the following: former smoker, alcohol or substance abuse or other Diagnostic and Statistical Manual of Mental Disorders, Fourth Edition (DSM-IV) Axis 1 psychiatric diagnosis within previous 6 months, lifetime alcohol or substance dependence (DSM-IV), non-German origin, no-mother-tongue level German, pregnancy, any medical condition that may interfere with the study (like serious vision or hearing impairment), central nervous system (CNS)-relevant medication within previous 6 months and CNS-relevant (neurological) illnesses (lifetime).

Inclusion/exclusion criteria were assessed through a medical examination, the Structured Clinical Interview for DSM-IV Axis I Disorders (SCID-1), questionnaires, drug screen, alcohol testing and CO measurement. This screening procedure resulted in the exclusion of $55 \%$ of all invited subjects. Given the various inclusion/exclusion criteria and given that the entire investigation of each proband was time-consuming ( 8 hours), no higher recruitment/study inclusion rates were realistically expected. In total, 2396 subjects were enrolled in the study. As of the intermediate phenotype approach of the study, only current smokers and never smokers ( $n=2381)$ were included to minimize phenotypic heterogeneity. Additionally, 67 of those had missing information in at least some items of the Pittsburgh Sleep Quality Index (PSQI) and were excluded, resulting in a study population of 2314 subjects.

\section{Instruments}

To evaluate sleep quality in smokers and non-smokers, the PSQI (Buysse et al. 1989) was used. Although this instrument was not specifically designed for the assessment of insomnia, it discriminates poor from good sleepers well and is an excellent general screening measure of sleep disturbances. This validated self-rating questionnaire gathers information in seven domains including subjective sleep quality, sleep latency, sleep duration, habitual sleep efficiency, sleep disturbances, use of sleep medication and daytime dysfunction over a period of 4 weeks. We used a slightly modified version assessing the same questions over a 2-week interval (Riemann \& Backhaus 1996). A component score of 0 is considered undisturbed, whereas a score of 3 is considered as severe subjective impairment. Taken together, these component scores build a global score ranging from 0, no impairment, to 21, severe disturbance of sleep. A global score of more than 5 is considered to represent disturbed sleep.

In addition to the above described inclusion criteria for the group 'smoker', the intensity of smoking and level of ND was determined by a number of additional scores. To determine severity of ND, the German version of the Fagerström questionnaire was applied (Bleich, HavemannReinecke \& Kornhuber 2002). The Fagerström Test (questionnaire) of Nicotine Dependence (FTND) is a selfreport instrument with six items rated either from 0 to 1 or from 0 to 3 (depending on the question) that can yield a total score of 10 , with higher scores indicating greater dependence. We divided the group of smokers into light smokers (LSs: FTND $\leq 4$ ) and heavy smokers (HSs: FTND $\geq 5$ ). As a behavioural proxy measure for ND, total number of daily cigarettes was asked for. As a biological marker of smoking intensity, plasma cotinine levels and exhaled CO were determined around 4:15 PM (Lindenberg et al. 2011). To determine smoking urges including nicotine craving smokers were assessed via self-report with the German version of the Questionnaire of Smoking Urges (QSU) (Tiffany \& Drobes 1991; Müller et al. 2001). The 32 QSU items form a summed total score that ranges from 32 to 224. As a lifetime measure for tobacco consumption, pack years were calculated. This measure, which we corrected for lifetime periods of abstinence, was chosen to determine a possible association between accumulated tobacco consumption and sleep disturbance. 
Table 1 Baseline demographics of participants.

\begin{tabular}{lccc}
\hline & Smokers & Non-smokers & $P$ \\
\hline Mean age, year (SD) & $36.8(12.8)$ & $34.7(13.3)$ & $\leq 0.001^{\mathrm{b}}$ \\
BMI (median/IQR) & $23.8(21.4-27.2)$ & $23.1(21.2-26.0)$ & $\leq 0.001^{\mathrm{b}}$ \\
Sex (\% males) & $45.2 \%$ & $39.4 \%$ & $\leq 0.005^{\mathrm{a}}$ \\
Lower education (less than 10 years of schooling) (\%) & $13.5 \%$ & $4.7 \%$ & $\leq 0.001^{\mathrm{a}}$ \\
Lower household income per year (€30.000 or less) (\%) & $74.3 \%$ & $62.0 \%$ & $\leq 0.001^{\mathrm{a}}$ \\
Beck Depression Inventory (18 points or more) (\%) & $2.8 \%$ & $0.7 \%$ & $\leq 0.001^{\mathrm{a}}$ \\
Alcohol Use Disorders Identification Test (8 points or more) (\%) & $18.5 \%$ & $6.3 \%$ & $\leq 0.001^{\mathrm{a}}$ \\
Years of studies or apprenticeship (3 years or less) (\%) & $63.4 \%$ & $50.7 \%$ & $\leq 0.001^{\mathrm{a}}$ \\
Attention deficit hyperactivity disorder symptomatology (median points/IQR) & $6(3-10)$ & $4(2-7)$ & $\leq 0.001^{\mathrm{b}}$ \\
State Trait Anxiety Inventory 1 (STAI1) (median/IQR) & $34(29-39)$ & $32(28-38)$ & $\leq 0.001^{\mathrm{b}}$ \\
STAI2 (median/IQR) & $34(29-41)$ & $33(28-40)$ & $\leq 0.004^{\mathrm{b}}$ \\
Perceived Stress Scale (median/IQR) & $20(15-25)$ & $19(14-23)$ & $\leq 0.001^{\mathrm{b}}$ \\
\hline
\end{tabular}

achi-quadrant test. ${ }^{\mathrm{b}}$ Mann-Whitney test. BMI = body mass index; IQR = interquartile range; SD = standard deviation.

A number of factors are known to be related to the occurrence of sleep disturbance such as age, sex, socioeconomic status and psychiatric co-morbidity (Ohayon 2002) that may have an influence on the observed associations between smoking status and the occurrence of sleep disturbances measured with the PSQI questionnaire. Therefore, the corrected odds ratios (ORs) for the prevalence of pathological scores after controlling for the possible confounders were calculated by multiple regression analysis.

As parameters possibly associated with the elevated prevalence of sleep disturbance in smokers, age (Zilli, Ficca \& Salzarulo 2009), sex (Buysse et al. 1989), body mass index (BMI), level of education (Grandner et al. 2010) and income, depressive symptomatology [Beck Depression Inventory (BDI) (Beck \& Steer 1987; Hautzinger et al. 1994)], anxiety [State Trait Anxiety Inventory (STAI) (Spielberger, Gorsuch \& Lushene 1970), German version by Laux et al. (1981)], ADHD symptomatology [DSM-IV Checklist ADHD (American Psychiatric Association 1994)], alcohol drinking behaviour [Alcohol Use Disorders Identification Test (AUDIT) (Babor et al. 1992)] and perceived stress [Perceived Stress Scale (PSS) (Cohen, Kamarck \& Mermelstein 1983)] were included into the model. Level of education was determined as years of school education and (average yearly household) income was categorized as less than $€ 10000$, €10 000-20 000, €20 000-30 000, €30 000-40 000, $€ 40$ 000-50 000, €50 000-60000 and more than $€ 60000$ p.a.

\section{Statistical analysis}

Data were analyzed using PASW Statistics 18 (SPSS Inc., Chicago, IL, USA). As descriptive measures, the percentage of smokers and never smokers who demonstrated problematic PSQI component scores of 2 or more and a global score of above 5 were calculated. A global score of more than 5 is considered as disturbed sleep quality. Single item scores of above 2 represent clinically relevant impairment of the respective domain including bad subjective sleep quality, sleep latency above 30 minutes and sleep duration of less than 6 hours (Buysse et al. 1989). To further analyse the nature of the relationship between smoking and variables of sleep quality, ORs and $95 \%$ confidence intervals (CIs) were determined. To control for the confounding effect of age, BMI, sex, education, income, depression, anxiety, alcohol consumption, hyperactivity and perceived stress, these factors were integrated in multiple regression analysis, and corrected ORs and 95\% CIs for the frequency of sleep disturbance in smokers in comparison to never smokers are presented. To analyse the influence of degree of ND, smoking urges, exhaled CO, number of cigarettes, pack years and plasma cotinine levels on sleep parameters, smokers were divided into lightly (FTND $\leq 4$ ) and heavily dependent (FTND $\geq 5$ ). For the other variables, the group of smokers was divided into halves according to lower and higher values of the respective parameter and $\chi^{2}$ tests were calculated to determine statistical significance of the results. To determine correlations of the measures of ND and craving for smokers, Spearman's rank correlations were calculated.

\section{RESULTS}

The study sample consisted of $N=1071$ currently smoking and $N=1243$ never smoking participants. Baseline demographics are displayed in Table 1. As expected in such a large sample of smokers and non-smokers, statistically significant differences in the degree of, frequency of or average of age (in years), BMI, sex, education (less than 10 years of schooling), income (equal or lower than $€ 30000$ per year), BDI (equal or more than 18 points), 
Table 2 Prevalence of sleep disturbance in smokers and non-smokers: uni-variate and multi-variate analyses.

\begin{tabular}{|c|c|c|c|c|c|c|}
\hline \multirow[b]{2}{*}{$P S Q I$} & $\begin{array}{l}\text { Percentage of smokers } \\
(n=1243)\end{array}$ & $\begin{array}{l}\text { Percentage of non- } \\
\text { smokers }(n=1071)\end{array}$ & OR $(95 \% C I)$ & Sign. $(P)$ & OR $(95 \% C I)$ & Sign. $(P)$ \\
\hline & \multicolumn{4}{|l|}{ Uni-variate analysis } & \multicolumn{2}{|c|}{ Multiple regression $* n=2314$} \\
\hline GS $>5$ & $28.1 \%$ & $19.1 \%$ & $1.66(1.37-2.02)$ & $<0.0001$ & $1.35(1.06-1.73)$ & 0.016 \\
\hline $\mathrm{SSQ} \geq 2$ & $15.8 \%$ & $10.1 \%$ & $1.68(1.31-2.15)$ & $<0.0001$ & $1.230 .90-1.69)$ & 0.194 \\
\hline $\mathrm{SL} \geq 2$ & $22.1 \%$ & $15.3 \%$ & $1.58(1.28-1.95)$ & $<0.0001$ & $1.42(1.09-1.84)$ & 0.009 \\
\hline $\mathrm{SD} \geq 2$ & $16.7 \%$ & $7.0 \%$ & $2.67(2.03-3.50)$ & $<0.0001$ & $1.98(1.45-2.71)$ & $<0.0001$ \\
\hline $\mathrm{SE} \geq 2$ & $7.2 \%$ & $6.1 \%$ & $1.19(0.86-1.65)$ & 0.299 & $0.83(0.54-1.26)$ & 0.371 \\
\hline S Dist $\geq 2$ & $7.7 \%$ & $5.1 \%$ & $1.57(1.12-2.21)$ & 0.008 & $1.14(0.75-1.73)$ & 0.554 \\
\hline $\mathrm{USM} \geq 2$ & $1.4 \%$ & $0.8 \%$ & $1.75(0.78-3.92)$ & 0.167 & $1.25(0.48-3.23)$ & 0.644 \\
\hline D Dys $\geq 2$ & $11.5 \%$ & $10.5 \%$ & $1.10(0.85-1.43)$ & 0.468 & $0.66(0.47-0.94)$ & 0.020 \\
\hline
\end{tabular}

*Adjusted for age (in years), sex, BMI, education (less/equal or more than 10 years of schooling), income (lower or equal/more than $€ 30.000$ per year), Beck Depression Inventory (less/equal or more than 18 points), Alcohol Use Disorders Identification Test (less/equal or more than 8 points), years of studies or apprenticeship, attention deficit hyperactivity disorder symptomatology, State Trait Anxiety Inventory 1 and 2 and Perceived Stress Scale. BMI $=$ body mass index; $\mathrm{CI}$ = confidence interval; D Dys = daytime dysfunction; GS = global score; OR = odds ratio; PSQI = Pittsburgh Sleep Quality Index; $\mathrm{SD}=$ sleep duration; $\mathrm{S}$ Dist = sleep disturbance; $\mathrm{SE}$ = sleep efficiency; $\mathrm{SL}$ = sleep latency; $\mathrm{SSQ}$ = subjective sleep quality; USM = use of sleep medication.

Table 3 Results of Spearman's rank correlations of the measures of nicotine dependence and craving for smokers.

\begin{tabular}{|c|c|c|c|c|c|c|}
\hline & FTND & QSU & $\begin{array}{l}\text { Cigarettes } \\
\text { per day }\end{array}$ & $\begin{array}{l}\text { Exhaled CO } \\
\text { (p.p.m.) }\end{array}$ & Cotinine & $\begin{array}{c}\text { Pack years } \\
\left(p y \_30 p 5\right)\end{array}$ \\
\hline FTND & & $0.604^{* *}$ & $0.838^{* *}$ & $0.685^{* *}$ & $0.668^{* *}$ & $0.608^{* *}$ \\
\hline QSU & & & $0.595^{* *}$ & $0.406^{* *}$ & $0.390^{* *}$ & $0.372^{* *}$ \\
\hline Cigarettes per day & & & & $0.705^{* *}$ & $0.685^{* *}$ & $0.704^{* *}$ \\
\hline Exhaled CO (p.p.m.) & & & & & $0.735^{* *}$ & $0.565^{* *}$ \\
\hline Cotinine & & & & & & $0.561^{* *}$ \\
\hline
\end{tabular}

${ }^{* *} P<0.01$; degree of smoking urges, low or high number of cigarettes smoked per day, concentration of exhaled CO (p.p.m.), plasma cotinine and pack years as a measure of accumulated cigarette consumption. FTND = Fagerström Test of Nicotine Dependence; QSU = Questionnaire of Smoking Urges.

AUDIT (equal or more than 8 points), 3 years or less of studies or apprenticeship, ADHD symptomatology, STAI1, STAI2 and PSS were observed. Significantly more smokers than non-smokers demonstrated overall poor sleep quality represented by a PSQI global score of over 5 (Table 2). Single component scores $\geq 2$ were observed more frequently in smokers compared with non-smokers for subjective sleep quality, increased sleep latency, a reduced sleep duration of less than 6 hours and sleep disturbance (Table 2). No significant difference was found for sleep efficiency, use of sleep medication and daytime dysfunction. Accordingly, the average PSQI component scores showed significantly higher values in smokers compared with non-smokers concerning subjective sleep quality $(0.89 \pm 0.67$ versus $0.77 \pm 0.64 ; P<0.001)$, sleep latency $(0.89 \pm 0.89$ versus $0.74 \pm 0.80 ; P<$ $0.001)$, sleep duration $(0.70 \pm 0.86$ versus $0.45 \pm$ $0.67 ; P<0.001)$, daytime dysfunction $(0.76 \pm 0.67$ versus $0.69 \pm 0.69 ; \quad P<0.005)$ and global score $(4.55 \pm 2.78$ versus $3.85 \pm 2.50 ; P<0.001)$. Nonsignificant differences were observed for sleep efficiency $(0.32 \pm 0.66$ versus $0.28 \pm 0.65 ; P=0.099)$, sleep disturbance $(0.91 \pm 0.51$ versus $0.89 \pm 0.46 ; P=0.413)$ and use of sleep medication $(0.05 \pm 0.30$ versus $0.04 \pm 0.25 ; P=0.260)$. In our sample, several confounders were significantly associated with PSQI items. After adjustment for age (in years), BMI, sex, education (less versus equal or more than 10 years of schooling), income (lower or equal versus more than $€ 30.000$ per year), BDI (less versus equal or more than 18 points), AUDIT (less versus equal or more than 8 points), years of studies or apprenticeship, ADHD, STAI1, STAI2 and PSS smokers demonstrated a significantly elevated OR for disturbed sleep in the domains sleep latency, sleep duration and global sleep quality and a reduced OR for daytime dysfunction (Table 2).

\section{Intensity of ND, smoking and sleep}

Measures of ND intensity significantly correlated with each other (Table 3). For further analysis of the influence of different measures of intensity of smoking and severity of $\mathrm{ND}$, we divided the group of smokers into LSs (FTND $\leq 4)$ and HSs (FTND $\geq 5)$, low $(\leq 100)$ and high $(>100)$ smoking urges, low $(\leq 11)$ and high $(>11)$ exhaled CO (p.p.m.), low (0-13) and high $(\geq 14)$ daily cigarette consumption and low $(\leq 92)$ and high $(>92)$ 
Table 4 Degree of nicotine dependence and craving and its association with sleep disturbance in smokers.

\begin{tabular}{|c|c|c|c|c|c|c|c|c|}
\hline \multirow[b]{2}{*}{ Smoking variable } & \multicolumn{8}{|c|}{ Percentage of subjects reporting elevated PSQI scores } \\
\hline & Global score $>5$ & $S S Q \geq 2$ & $S L \geq 2$ & $S D \geq 2$ & $S E \geq 2$ & $S$ Dist $\geq 2$ & $U S M \geq 2$ & $D$ Dys $\geq 2$ \\
\hline \multicolumn{9}{|l|}{ FTND } \\
\hline $\begin{array}{l}\text { Light smokers (FTND } \leq 4) \\
\quad n=740(\%)\end{array}$ & 26.5 & 15.3 & 20.0 & 13.1 & 6.6 & 6.5 & 1.5 & 11.5 \\
\hline $\begin{array}{l}\text { Heavy smokers }(\mathrm{FTND} \geq 5) \\
\quad n=325(\%)\end{array}$ & 31.7 & 17.2 & 27.1 & 24.9 & 8.6 & 10.8 & 1.5 & 11.4 \\
\hline$P$ & 0.082 & 0.420 & 0.010 & $<0.0001$ & 0.247 & 0.016 & 0.949 & 0.962 \\
\hline \multicolumn{9}{|l|}{ QSU } \\
\hline Low $[\leq 100 ; n=547(\%)]$ & 26.2 & 13.9 & 19.2 & 15.0 & 7.1 & 6.8 & 1.1 & 10.6 \\
\hline High $[>100 ; n=500(\%)]$ & 30.2 & 17.6 & 24.8 & 18.4 & 7.4 & 8.8 & 1.6 & 12.4 \\
\hline$P$ & 0.130 & 0.106 & 0.030 & 0.158 & 0.905 & 0.247 & 0.805 & 0.383 \\
\hline \multicolumn{9}{|l|}{ Cigarettes per day } \\
\hline $0-13 ; n=547(\%)$ & 25.4 & 15.4 & 19.6 & 10.6 & 6.4 & 6.6 & 1.8 & 12.4 \\
\hline $14+; n=524(\%)$ & 30.9 & 16.2 & 24.8 & 23.1 & 8.0 & 9.0 & 1.0 & 10.5 \\
\hline$P$ & 0.045 & 0.698 & 0.039 & $<0.0001$ & 0.306 & 0.144 & 0.224 & 0.321 \\
\hline \multicolumn{9}{|l|}{ Exhaled CO (p.p.m.) } \\
\hline Low $(\leq 11) ; n=512(\%)$ & 27.3 & 15.6 & 20.9 & 12.7 & 6.8 & 8.2 & 1.8 & 14.3 \\
\hline High $(>11) ; n=483(\%)$ & 29.4 & 16.6 & 24.2 & 22.2 & 7.7 & 7.9 & 1.0 & 7.7 \\
\hline$P$ & 0.482 & 0.730 & 0.225 & $<0.0001$ & 0.627 & 0.907 & 0.423 & 0.001 \\
\hline \multicolumn{9}{|l|}{ Cotinine } \\
\hline Low $(\leq 92) ; n=513(\%)$ & 30.2 & 17.0 & 21.1 & 12.5 & 9.4 & 8.4 & 1.4 & 14.4 \\
\hline High $(>92) ; n=494(\%)$ & 26.7 & 14.2 & 24.1 & 20.4 & 5.1 & 7.5 & 1.4 & 9.1 \\
\hline$P$ & 0.235 & 0.225 & 0.259 & 0.001 & 0.010 & 0.642 & 1.000 & 0.011 \\
\hline \multicolumn{9}{|l|}{ Pack years } \\
\hline$\leq$ age adjusted mean, $n=541(\%)$ & 26.6 & 14.2 & 20.9 & 12.6 & 7.0 & 5.9 & 1.7 & 11.3 \\
\hline$>$ age adjusted mean, $n=485(\%)$ & 30.5 & 17.3 & 24.1 & 21.2 & 7.6 & 9.9 & 1.2 & 11.8 \\
\hline$P$ & 0.188 & 0.197 & 0.231 & $<0.001$ & 0.720 & 0.020 & 0.613 & 0.845 \\
\hline $\begin{array}{l}P \text { (additionally adjusted for } \\
\text { cigarettes per day) }\end{array}$ & 0.587 & 0.298 & 0.892 & 0.277 & 0.820 & 0.062 & 0.896 & 0.589 \\
\hline
\end{tabular}

Percentage of smokers with a component score of $\geq 2$ corresponding to a higher level of disturbance in the areas. Degree of smoking urges, low or high number of cigarettes smoked per day, concentration of exhaled CO (p.p.m.), plasma cotinine and pack years as a measure of accumulated cigarette consumption. Missing values for different reasons included questionnaires filled out incorrectly, data not collected, data quality, etc. However, we have checked for distinct patterns within missing values (center dependence, etc.) and did not find an explanatory pattern. D Dys = daytime dysfunction depending on degree of nicotine dependence; FTND = Fagerström Test of Nicotine Dependence; QSU = Questionnaire of Smoking Urges; SD = sleep duration; S Dist = sleep disturbance; $\mathrm{SE}=$ sleep efficiency; $\mathrm{SL}=$ sleep latency; $\mathrm{SSQ}=$ subjective sleep quality; USM = use of sleep medication.

cotinine concentrations. Consistently, higher intensity of ND, exhaled CO (p.p.m.), number of cigarettes and cotinine levels was associated with a higher frequency of an elevated score of the PSQI-item sleep duration, corresponding to shorter sleep in these subjects (Table 4, Fig. 1). However, the degree of smoking urges was not significantly associated with sleep duration. Additionally, a higher degree of ND and a higher intensity of smoking urges as well as an elevated number of cigarettes per day were associated with a higher frequency of impaired sleep latency. An increased prevalence of elevated sleep disturbance was only observed in the heavily dependent smokers, i.e. the more severely dependent smokers demonstrated a trend towards a higher percentage of PSQI global scores above 5. Additionally, a significant association between number of cigarettes per day and overall poor sleep quality represented by a global score above 5 was observed with worse sleep demonstrated by HSs.
Furthermore, severe daytime dysfunction was less frequent in smokers demonstrating high levels of exhaled $\mathrm{CO}$ and cotinine. Higher pack years, adjusted for age, were associated with a higher frequency of short sleep duration and a higher percentage of sleep disturbance. However, additionally adjusting for the number of cigarettes currently smoked, no significant association between accumulated nicotine consumption and any sleep item was observed.

\section{DISCUSSION}

To the best of our knowledge, this is the first investigation to report on subjective sleep quality in healthy smokers without medical or psychiatric co-morbidity in comparison to never smokers using a validated sleep questionnaire. In this large case-control study excluding co-morbid conditions such as depression, alcohol 

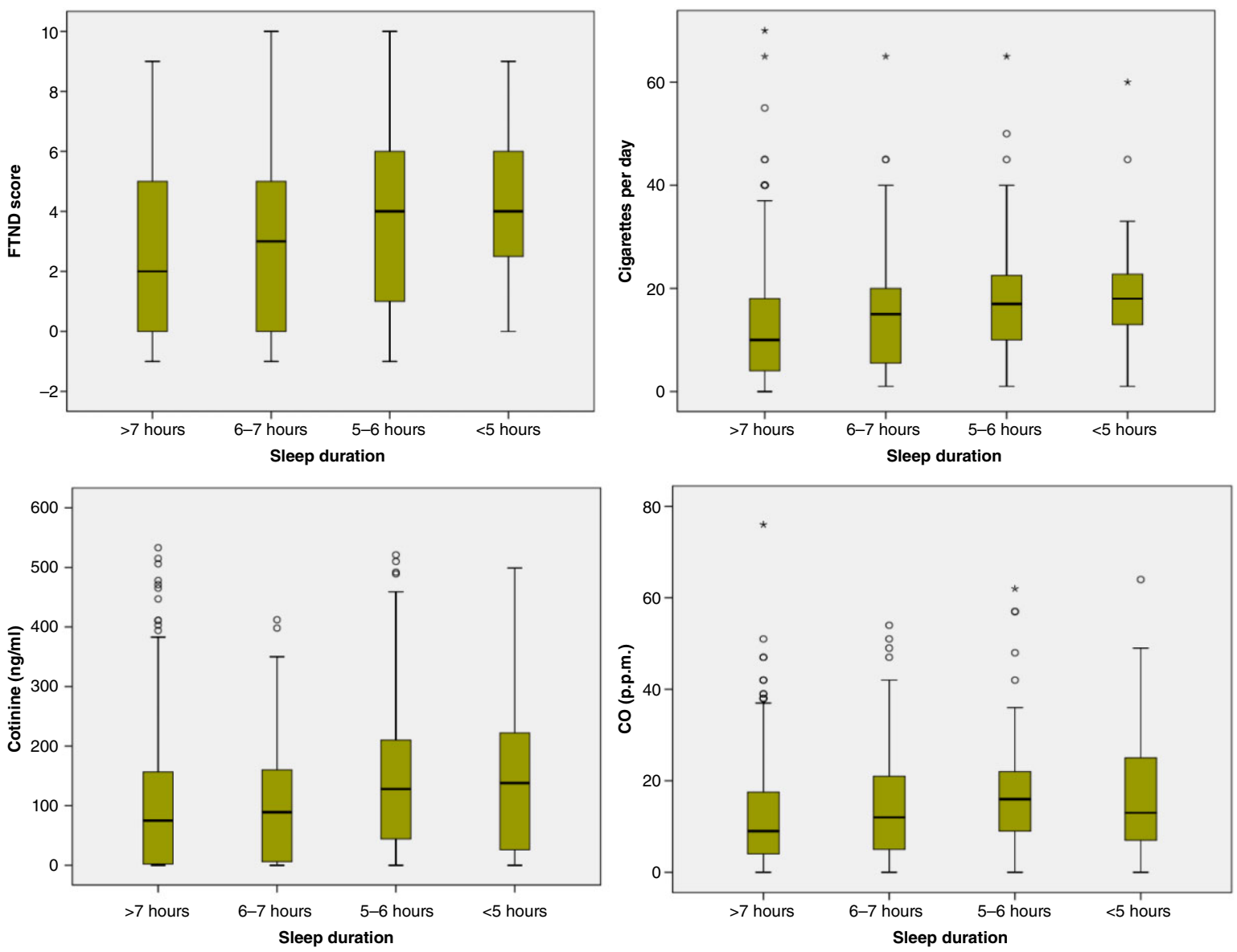

Figure I Sleep duration and measures of smoking intensity. Box plots of distribution of nicotine dependence scores, number of cigarettes smoked per day, exhaled CO (p.p.m.) and cotinine plasma levels in the groups of smokers sleeping more than 7 hours, 6-7 hours, 5-6 hours and less than 5 hours. Asterisks are extreme outliers. FTND = Fagerström Test of Nicotine Dependence

addiction or abuse and other active psychiatric disorders, smokers were found to suffer from significantly affected sleep quality in several domains. Smokers demonstrated considerably more often than never smokers elevated scores in the domains subjective sleep quality, increased sleep latency, reduced sleep duration of less than 6 hours, sleep disturbance and an overall reduced sleep quality represented by a PSQI global score of above 5. After adjusting for age, BMI, education, income, depressiveness, alcohol consumption, attention deficit hyperactivity symptoms, anxiety and perceived stress, smokers still had a higher risk to demonstrate impaired sleep latency, sleep duration and overall poor sleep quality but demonstrated a lower risk for daytime dysfunction.

Although to date no other study has used a validated questionnaire integrating several aspects of sleep to one global score describing the overall sleep quality, the significantly elevated prevalence of PSQI scores above 5 observed in our smokers may at least in part correspond to findings of more general symptoms of insomnia such as non-restorative sleep, difficulties waking up (Wetter \& Young 1994), more frequent problems with staying asleep and increased daytime sleepiness (Phillips \& Danner 1995), increased ORs for insomnia (Riedel et al. 2004) and subjectively insufficient sleep (Kaneita et al. 2005) in smokers described by others. More specifically, the finding of a disturbed sleep latency with a higher number of smokers reporting an increased time to fall asleep is in accordance with studies reporting smokers to have more difficulties getting to sleep (Wetter \& Young 1994; Phillips \& Danner 1995; Kaneita et al. 2005). Likewise, an elevated OR for a short sleep duration has been described in Japanese smoking pregnant women (Kaneita et al. 2005). A reduction of about 30 minutes in sleep duration was observed by Palmer et al. (1980) in males and females and by 24 minutes in LSs in a random sample sleep diary study by Riedel et al. (2004).

Adjusting for the confounders, age, BMI, sex, education, income, depression, alcohol consumption, attention deficit hyperactivity symptoms, anxiety and perceived stress appeared to reduce only slightly the strength of association between smoking and the occurrence of an impaired sleep latency, sleep duration and global poor sleep quality. Therefore, direct smoking-related aspects 
seem to have a strong effect on these parameters of sleep quality. The lower risk of demonstrating daytime dysfunction in smokers after adjusting for these confounders may be related to the activating properties of nicotine consumed by the smokers antagonizing sleepiness and lack of enthusiasm. This interpretation is supported by the finding that the smokers with a higher concentration of cotinine and exhaled $\mathrm{CO}$, reflecting a higher intake of nicotine bearing smoke, demonstrate a lower rate of daytime dysfunction.

\section{Intensity of ND, smoking and smoking urges}

To further explore the observed relationship between smoking and sleep, we investigated the influence of several markers of smoking intensity, including ND, a measure that has not been studied before. In our sample, we found a significantly worse sleep latency, sleep duration and sleep disturbance as well as a trend towards a decreased global sleep quality in highly dependent smokers compared with lightly dependent smokers measured by the FTND. A comparable pattern of sleep disturbance was observed for high and low amounts of cigarettes smoked daily. This may well be explained by the strong correlation between FTND and cigarettes per day found in this study which can be interpreted in the way that cigarettes per day is a useful proxy measure for the degree of ND (Lindenberg et al. 2011). An association between high versus low levels of exhaled CO and cotinine was found only for sleep duration with higher levels being associated with a higher percentage of smokers sleeping less than 6 hours. The weaker association of these two parameters with measures of sleep quality is in accordance with less strong correlations of FTND on one hand and exhaled CO and plasma cotinine plasma levels on the other hand (Lindenberg et al. 2011). The less frequently observed daytime dysfunction in smokers with high levels of exhaled $\mathrm{CO}$ and cotinine may be a result of the activating aspects of nicotine. In addition to exploring the association of acute effects of smoking and sleep, we analysed the influence of accumulated amount of cigarettes smoked during the lifetime of our smokers on PSQI items. After adjusting for age, high pack years appeared to be associated with a significantly higher percentage of subjects sleeping less than 6 hours. However, after further adjusting for the currently smoked number of cigarettes per day, this association was lost. Therefore, the originally observed association is most likely due to acute effects of nicotine consumption and most likely not a result of the accumulated nicotine consumption or accumulated toxic effects.

Remarkably, no significant influence of degree of smoking urges on any parameter of sleep quality other than sleep latency was observed. This may be explained by the circumstance that questionnaires were filled out only shortly after the last cigarette, which most likely was associated with lower QSU values (Müller et al. 2001) and possibly a lower variability of smoking urges. Although some of the sleep disturbance of HSs has been related to nicotine craving in an earlier descriptive study by Rieder et al. (2001), to the best of our knowledge no other systematic study on the influence of the degree of craving on sleep in smokers exists. However, in treatment-seeking alcohol-dependent patients, the degree of craving appeared to be associated with sleep problems, although after controlling for mood disturbance no such relationship was found anymore (Chakravorty et al. 2010). Therefore, the lack of an association of degree of smoking urges with sleep parameters in our sample may be due to the rigorous exclusion criteria, minimizing the degree of mood disturbance in our sample. In that respect, our findings are similar in that there may not exist a direct, mood-independent relationship between craving and sleep quality, neither in alcohol nor ND.

The most consistent finding for an association between parameters of smoking intensity and sleep in our study was found for a reduction in sleep duration. To our knowledge, there are no other studies investigating FTND scores, exhaled CO or cotinine plasma levels and sleep duration. Comparable results for number of cigarettes and sleep duration have been reported by Kaneita et al. (2005). Heavily in comparison to lightly smoking pregnant women had an even higher OR to suffer from short sleep duration in comparison to non-smokers (Kaneita et al. 2005). However, another investigation found somewhat contradictory results. Although a reduced sleep duration was observed in LSs in comparison to non-smokers, this was not true for HSs in that random sample sleep diary study (Riedel et al. 2004).

A number of hypotheses have been proposed for the association between cigarette smoking and poor sleep, like increased catecholamine output, general unhealthy approach to life-style, more disturbed personality type and the stimulant effects of smoke ingredients (Phillips \& Danner 1995). The consistent relationship between measures of smoking intensity and sleep duration in our sample suggests that there may be a direct pharmacological effect of smoking on sleep disturbance. The most likely biochemical candidate to cause sleep disturbance is nicotine. Investigating the effects of nicotine in non-smoking healthy subjects, some authors found sleep duration to be reduced by about 30-50 minutes under the application of a 24-hour nicotine patch (Davila et al. 1994; SalinPascual et al. 1995), others however were not able to confirm this finding (Gillin et al. 1994). Additionally, considering the relatively short half-life of nicotine (1-2 hours) (Benowitz et al. 1982), the influence of nicotine administered through a patch delivering nicotine while asleep may have different effects than nicotine reaching the body via a cigarette before bedtime. 
Another way of thinking about smoking and sleep comes from the other side, interpreting the higher number of cigarettes smoked as a result rather than a cause of the associated reduced sleep duration. Firstly, a reduced time asleep increases the opportunity to smoke while awake. However, it is more likely that a reduced sleep duration may originate from other smoking-independent life-style aspects exhibited by the smokers. Following this line of thought, the higher number of cigarettes smoked may be related to the sleepiness resulting from a reduction in total sleep time and may be intended to antagonize the unwanted consequences of lack of sleep. This interpretation is supported by the finding of a lower frequency of daytime dysfunction in the group of smokers with higher cotinine levels. In the PSQI questionnaire, daytime dysfunction corresponds to the two items: tiredness and lack of energy. The higher levels of cotinine reflect an increased consumption of the stimulant nicotine, thereby effectively antagonizing the assumable tiredness. Additionally, this view is endorsed by a recent study on experimentally induced sleep loss. In that study, sleep loss was demonstrated to be followed by an increase in number of smoked cigarettes (Hamidovic \& de Wit 2009). These two factors, smoking to antagonize daytime sleepiness and the shortlived residual effects of nicotine consumption on sleep, may actually build up to a vicious circle, where cigarette smoking is arousing the individual who then sleeps less and consecutively smokes more the following day to possibly compensate for the effects of sleep loss on factors such as cognitive and emotional consequences like irritability, impairment of attention and inhibitory control.

\section{Relevance of sleep disturbance and shortened sleep}

The reduction in sleep duration may be of direct relevance for health issues of smokers. Over the last decade, sleep disturbance, especially a reduction in sleep duration, has been identified to play an important role in the pathophysiology of a number of physical as well as mental disorders and to be related to mortality. A recent meta-analysis including 16 studies with more than 1.3 million participants came to the conclusion that short sleep duration is associated with a significantly increased risk of death (Cappuccio et al. 2010). In one study, short sleep duration of less than 6 hours, as found in $17 \%$ of our smoking sample in comparison to $7.2 \%$ of the nonsmokers, was found to increase mortality by a factor of up to 2.4 in men compared with those with 7-7.9 hours of sleep (Amagai et al. 2004).

Other studies linked short sleep duration to an increased risk for the development of specific disorders such as obesity (Cappuccio et al. 2008), hypertension (Vgontzas et al. 2009), diabetes type II (Vgontzas et al. 2009), the susceptibility to catch a common cold (Cohen et al. 2009) and insomnia to an elevated risk to develop depression (Baglioni et al. 2011) and addictive behaviour (Breslau et al. 1996). Some of the increase in mortality observed in smokers, therefore, may be related to their shorter sleep duration, and consequently the reduction of mortality risk observed after smoking cessation (Doll et al. 2004) may partly originate from the parallel improvement of sleep quality and increase in sleep duration (Zhang et al. 2006).

This study has some limitations. Firstly, although impaired sleep quality is one of the criteria for insomnia as per the International Classification of Sleep Disorders, Second Edition (American Academy of Sleep Medicine 2005) and DSM-IV, it is a poorly defined construct. It is considered a complex phenomenon that is difficult to define and measure objectively (Buysse et al. 1989). Additionally, recent research shows that sleep quality may be a measure of daytime tiredness and the number of nocturnal awakenings (Harvey et al. 2008). However, the PSQI is a validated and widely used questionnaire considering a variety of aspects of sleep distinguishing well between sleep disturbed patients and controls using component scores as well as the global score (Buysse et al. 1989). Secondly, although we intended to accomplish population representativeness, due to the strict inclusion and exclusion criteria and the extensive time-consuming investigation of each proband, only a portion of the population could be recruited. This may reduce the generalizability of the results. However, e.g. excluding former smokers rather helped to minimize phenotypic heterogeneity which is relevant because some earlier studies could not find differences between former smokers and never smokers concerning sleep quality (Zhang et al. 2006). A further limitation is the restriction to subjective sleep data. Objective measures (e.g. polysomnographic/actigraphic data) of sleep quality including sleep duration or subjective sleep diary data could possibly have differed from the collected subjective data. Therefore, the likely relevance of the observed reduced subjective sleep quality should be confirmed by studies evaluating objective sleep duration in smokers and never smokers. However, DSM-IV and International Classification of Diseases, Tenth Revision criteria for insomnia are also restricted to subjective reports of the individual and this form of sleep disorder has been demonstrated to be a serious risk factor for further health sequelae. Hence, the observed differences in sleep quality and duration of smokers and never smokers are very likely of high relevance. Another limitation is that we have not screened the subjects for breathing-related sleep disorders that may be associated with symptoms of fragmented sleep and may have confounded the results of this study. However, we adjusted for BMI, an important risk factor for the occurrence of sleep-related breathing disorders. Therefore, it appears unlikely that breathing-related sleep disorders would explain the observed differences. The 
occurrence of subjects reporting an AUDIT score of 8 points or more is somewhat astonishing as subjects were screened to be free of Axis I disorders. The higher percentage of smokers versus non-smokers demonstrating scores of 8 points or more, however, is in line with the literature demonstrating higher alcohol consumption among smokers. This discrepancy may be related to the highly variable prevalence of conspicuous AUDIT scores in different populations ranging from $4 \%$ to $74 \%$ and its variable sensitivity and specificity depending on the population (Berner et al. 2007). However, we are confident that the AUDIT questionnaire was a useful tool to control for the degree of alcohol drinking behaviour in our analysis of the occurrence of sleep disturbance in smokers versus non-smokers. Furthermore, the exclusion of subjects suffering from Axis I psychiatric disorders in our study may restrict the generalizability of our results.

Despite these limitations, this study provides unique information on the association of smoking and sleep quality. For the first time, a broad range of measures of ND and intensity of smoking were identified to be related to measures of sleep quality, especially sleep duration. Further research should explore the precise mechanisms involved in the decrease in sleep quality and reduction of sleep duration to better understand ND and the pathophysiology of the related sleep disturbance. The increased understanding of these mechanisms may improve intervention and optimize smoking cessation programs. Additionally, this knowledge may provide a solid basis to analyse and disclose the genetic mechanisms affecting both behaviours and their relationship.

\section{FUNDING}

The study 'Genetics of Nicotine Dependence and Neurobiological Phenotypes' (Wi1316/9-1—Principal Investigator: GW) was funded by the German Research Foundation (Deutsche Forschungsgemeinschaft) as part of the Priority Program (Schwerpunktprogramm) SPP1226: 'Nicotine-Molecular and Physiological Effects in CNS' (Coordinator: GW) (http://www.nicotineresearch.de).

\section{Authors Contribution}

GW and SC were responsible for the study concept and design. AM, GG, ND, NT, JG, MW, JK and GW contributed to the acquisition of the data. TW and AD-L designed and supported the data bank. BS and UG performed the statistical analysis. FK performed the plasma analysis. SC and AR drafted the manuscript. DR, AJ and DK provided critical revision of the manuscript for important intellectual content. All authors critically reviewed content and approved final version for publication.

\section{References}

Aklin WM, Moolchan ET, Luckenbaugh DA, Ernst M (2009) Early tobacco smoking in adolescents with externalizing disorders: inferences for reward function. Nicotine Tob Res 11:750-755.

Amagai Y, Ishikawa S, Gotoh T, Doi Y, Kayaba K, Nakamura Y, Kajii E (2004) Sleep duration and mortality in Japan: the Jichi Medical School Cohort Study. J Epidemiol 14:124-128.

American Academy of Sleep Medicine (2005) International Classification of Sleep Disorders, 2nd edn. Diagnostic and Coding Manual ed. Westchester, IL: American Academy of Sleep Medicine.

American Psychiatric Association (1994) DSM IV Checklist Attention Deficit Hyperactivity Disorder. APA.

American Psychiatric Association (2000) American Psychiatric Association: The Diagnostic and Statistical Manual of Mental Disorders, 4th edn. text revision ed. Washington D.C.: American Psychiatric Association.

Babor TF, de la Fuente JR, Saunders J, Grant M (1992) AUDIT. The Alcohol Use Disorders Identification Test. Guidelines for Use in Primary Health Care. Geneva: World Health Organization.

Baglioni C, Battagliese G, Feige B, Spiegelhalder K, Nissen C, Voderholzer U, Lombardo C, Riemann D (2011) Insomnia as a predictor of depression: a meta-analytic evaluation of longitudinal epidemiological studies. J Affect Disord 135:10-19.

Beck A, Steer R (1987) Beck Depression Inventory-Manual. San Antonio, TX: The Psychological Corporation.

Benca RM, Obermeyer WH, Thisted RA, Gillin JC (1992) Sleep and psychiatric disorders. A meta-analysis. Arch Gen Psychiatry 49:651-668.

Benowitz NL, Jacob P, 3rd, Jones RT, Rosenberg J (1982) Interindividual variability in the metabolism and cardiovascular effects of nicotine in man. J Pharmacol Exp Ther 221:368372.

Berner MM, Kriston L, Bentele M, Harter M (2007) The alcohol use disorders identification test for detecting at-risk drinking: a systematic review and meta-analysis. J Stud Alcohol Drugs 68:461-473.

Bleich S, Havemann-Reinecke U, Kornhuber J (2002) Der Fagerström-Test für Nikotinabhängigkeit (FTNA). Göttingen, Bern, Toronto, Seattle: Hogrefe-Verlag.

Breslau N, Roth T, Rosenthal L, Andreski P (1996) Sleep disturbance and psychiatric disorders: a longitudinal epidemiological study of young adults. Biol Psychiatry 39:411-418.

Buysse DJ, Reynolds CF, 3rd, Monk TH, Berman SR, Kupfer DJ (1989) The Pittsburgh Sleep Quality Index: a new instrument for psychiatric practice and research. Psychiatry Res 28:193213.

Cappuccio FP, D’Elia L, Strazzullo P, Miller MA (2010) Sleep duration and all-cause mortality: a systematic review and meta-analysis of prospective studies. Sleep 33:585-592.

Cappuccio FP, Taggart FM, Kandala NB, Currie A, Peile E, Stranges S, Miller MA (2008) Meta-analysis of short sleep duration and obesity in children and adults. Sleep 31:619626.

Chakravorty S, Kuna ST, Zaharakis N, O'Brien CP, Kampman KM, Oslin D (2010) Covariates of craving in actively drinking alcoholics. Am J Addict 19:450-457.

Cohen S, Doyle WJ, Alper CM, Janicki-Deverts D, Turner RB (2009) Sleep habits and susceptibility to the common cold. Arch Intern Med 169:62-67. 
Cohen S, Kamarck T, Mermelstein R (1983) A global measure of perceived stress. J Health Soc Behav 24:385-396.

Cohrs S (2008) Sleep disturbances in patients with schizophrenia: impact and effect of antipsychotics. CNS Drugs 22:939962.

Colrain IM, Trinder J, Swan GE (2004) The impact of smoking cessation on objective and subjective markers of sleep: review, synthesis, and recommendations. Nicotine Tob Res 6:913925.

Davila DG, Hurt RD, Offord KP, Harris CD, Shepard JW, Jr (1994) Acute effects of transdermal nicotine on sleep architecture, snoring, and sleep-disordered breathing in nonsmokers. Am J Respir Crit Care Med 150:469-474.

Doll R, Peto R, Boreham J, Sutherland I (2004) Mortality in relation to smoking: 50 years' observations on male British doctors. BMJ 328:1519.

Elkins IJ, McGue M, Iacono WG (2007) Prospective effects of attention-deficit/hyperactivity disorder, conduct disorder, and sex on adolescent substance use and abuse. Arch Gen Psychiatry 64:1145-1152.

Gillin JC, Lardon M, Ruiz C, Golshan S, Salin-Pascual R (1994) Dose-dependent effects of transdermal nicotine on early morning awakening and rapid eye movement sleep time in nonsmoking normal volunteers. J Clin Psychopharmacol 14:264-267.

Grandner MA, Patel NP, Gehrman PR, Xie D, Sha D, Weaver T, Gooneratne N (2010) Who gets the best sleep? Ethnic and socioeconomic factors related to sleep complaints. Sleep Med 11:470-478.

Hamidovic A, de Wit H (2009) Sleep deprivation increases cigarette smoking. Pharmacol Biochem Behav 93:263269.

Harvey AG, Stinson K, Whitaker KL, Moskovitz D, Virk H (2008) The subjective meaning of sleep quality: a comparison of individuals with and without insomnia. Sleep 31:383393.

Hautzinger M, Bailer M, Worall H, Keller F (1994) Das BeckDepressions-Inventar (BDI). Huber: Bern.

$\mathrm{Hu}$ L, Sekine M, Gaina A, Kagamimori S (2007) Association between sleep quality and smoking in Japanese civil servants. Sleep Biol Rhythms 5:196-203.

Hughes JR, Higgins ST, Bickel WK (1994) Nicotine withdrawal versus other drug withdrawal syndromes: similarities and dissimilarities. Addiction 89:1461-1470.

Jaehne A, Loessl B, Barkai Z, Riemann D, Hornyak M (2009) Effects of nicotine on sleep during consumption, withdrawal and replacement therapy. Sleep Med Rev 13:363-377.

Kaneita Y, Ohida T, Takemura S, Sone T, Suzuki K, Miyake T, Yokoyama E, Umeda T (2005) Relation of smoking and drinking to sleep disturbance among Japanese pregnant women. Prev Med 41:877-882.

Klink M, Quan SF (1987) Prevalence of reported sleep disturbances in a general adult population and their relationship to obstructive airways diseases. Chest 91:540-546.

Krishnan-Sarin S, Reynolds B, Duhig AM, Smith A, Liss T, McFetridge A, Cavallo DA, Carroll KM, Potenza MN (2007) Behavioral impulsivity predicts treatment outcome in a smoking cessation program for adolescent smokers. Drug Alcohol Depend 88:79-82.

Laux L, Glanzmann P, Schaffner P, Spielberger CD (1981) Das State-Trait-Angstinventar. Theoretische Grundlagen und Handanweisung. Beltz: Weinheim.
Lindenberg A, Brinkmeyer J, Dahmen N, Gallinat J, de Millas W, Mobascher A, Wagner M, Schulze-Rauschenbach S, Grunder G, Spreckelmeyer KN, Clepce M, Thurauf N, von der Goltz C, Kiefer F, Steffens M, Holler D, Diaz-Lacava A, Wienker T, Winterer G (2011) The German multi-centre study on smokingrelated behavior-description of a population-based casecontrol study. Addict Biol 16:638-653.

Müller V, Mucha R, Ackermann K, Pauli P (2001) Die Erfassung des Cravings bei Rauchen mit einer deutschen Version des 'Questionnaire on Smoking Urges' (QSU-G). Z Klin Psychol Psychother 30:164-171.

Ohayon MM (2002) Epidemiology of insomnia: what we know and what we still need to learn. Sleep Med Rev 6:97-111.

O’Loughlin J, Karp I, Koulis T, Paradis G, Difranza J (2009) Determinants of first puff and daily cigarette smoking in adolescents. Am J Epidemiol 170:585-597.

Palmer CD, Harrison GA, Hiorns RW (1980) Association between smoking and drinking and sleep duration. Ann Hum Biol 7:103-107.

Peterson MJ, Benca RM (2006) Sleep in mood disorders. Psychiatr Clin North Am 29:1009-1032. abstract ix.

Phillips BA, Danner FJ (1995) Cigarette smoking and sleep disturbance. Arch Intern Med 155:734-737.

Riedel BW, Durrence HH, Lichstein KL, Taylor DJ, Bush AJ (2004) The relation between smoking and sleep: the influence of smoking level, health, and psychological variables. Behav Sleep Med 2:63-78.

Rieder A, Kunze U, Groman E, Kiefer I, Schoberberger R (2001) Nocturnal sleep-disturbing nicotine craving: a newly described symptom of extreme nicotine dependence. Acta Med Austriaca 28:21-22.

Riemann D, Backhaus J (1996) Therapy of Insomnia. Weinheim: Beltz/PUV.

Salin-Pascual RJ, de la Fuente JR, Galicia-Polo L, Drucker-Colin R (1995) Effects of transderman nicotine on mood and sleep in nonsmoking major depressed patients. Psychopharmacology (Berl) 121:476-479.

Spielberger CD, Gorsuch RL, Lushene RE (1970) STAI, Manual for the State-Trait Anxiety Inventory. Palo Alto, CA: Consulting Psychologist Press.

Tiffany ST, Drobes DJ (1991) The development and initial validation of a questionnaire on smoking urges. Br J Addict 86:1467-1476.

Trosclair A, Dube SR (2010) Smoking among adults reporting lifetime depression, anxiety, anxiety with depression, and major depressive episode, United States, 2005-2006. Addict Behav 35:438-443.

Tsuno N, Besset A, Ritchie K (2005) Sleep and depression. J Clin Psychiatry 66:1254-1269.

Vgontzas AN, Liao D, Pejovic S, Calhoun S, Karataraki M, Bixler EO (2009) Insomnia with objective short sleep duration is associated with type 2 diabetes: a population-based study. Diabetes Care 32:1980-1985.

Wetter DW, Young TB (1994) The relation between cigarette smoking and sleep disturbance. Prev Med 23:328-334.

Winterer G (2010) Why do patients with schizophrenia smoke? Curr Opin Psychiatry 23:112-119.

Zhang L, Samet J, Caffo B, Punjabi NM (2006) Cigarette smoking and nocturnal sleep architecture. Am J Epidemiol 164:529537.

Zilli I, Ficca G, Salzarulo P (2009) Factors involved in sleep satisfaction in the elderly. Sleep Med 10:233-239. 